\title{
Into the future with little past: Exploring mental time travel in a patient with
}

damage to the mammillary bodies/fornix

Jacqui Tedder ${ }^{1}$, Laurie Miller ${ }^{2,3}$, Sicong $\mathrm{Tu}^{2,4,5}$, Michael Hornberger ${ }^{2,4,6}$ and Suncica $\operatorname{Lah}^{1,2}$

${ }^{1}$ School of Psychology, University of Sydney, Sydney NSW Australia

${ }^{2}$ ARC Centre of Excellence in Cognition and its Disorders, Sydney NSW Australia ${ }^{3}$ Neuropsychology Unit, Royal Prince Alfred Hospital and Central Clinical School, University of Sydney, Sydney, NSW, Australia

${ }^{4}$ Neuroscience Research Australia, Sydney, NSW, Australia

${ }^{5}$ School of Medical Sciences, Faculty of Medicine, University of New South Wales, Sydney, NSW, Australia

${ }^{6}$ Norwich Medical School, University of East Anglia, Norwich, UK

Corresponding author: Suncica Lah, School of Psychology, Mungo Mac Callum Building (A18), The University of Sydney NSW 2006, Australia

Tel: +61-2-9351 2648, Fax: +61-2-9351 7328, e-mail: suncica.lah@sydney.edu.au 
Into the future with little past

Mailing addresses:

Jacqui Tedder, School of Psychology, Mungo Mac Callum Building (A18), The

University of Sydney NSW 2006, Australia, Tel: +61-2-9351 2648, e-mail:

jacqui_tedder@hotmail.com

Laurie Miller, Neuropsychology Unit, Royal Prince Alfred Hospital, Camperdown, NSW Australia 2050; Tel: 612-95157812; email: laurie.miller@sydney.edu.au

Sicong Tu, Neuroscience Research Australia, Barker St., Randwick, NSW, 2031, Australia, Tel: +61 029399 1025, e-mail: s.tu@neura.edu.au

Michael Hornberger, Norwich Medical School, University of East Anglia, Norwich, NR4 7TJ, UK, Tel: + 441603 593061, e-mail: m.hornberger@uea.ac.uk 
Into the future with little past

\begin{abstract}
Objective: Remembering the past and imaging the future are both manifestations of “mental time travel” (Tulving, 1985). These processes have been found to be impaired in patients with bilateral hippocampal lesions. Here we examined the question of whether future thinking is affected by other Papez circuit lesions, namely damage to the mammillary bodies/fornix.
\end{abstract}

Method: Case (SL) was a 43 year old woman who developed dense anterograde and retrograde amnesia suddenly, as a result of Wernicke-Korsakoff's syndrome. A region of interest volumetric MRI analysis was performed. We assessed past and future thinking in SL and 11 control subjects of similar age and education with the adapted Autobiographical Interview (AI). Participants also completed a battery of neuropsychological tests.

Results: Volumetric MRI analyses revealed severely reduced fornix and mammillary body volumes, but intact hippocampi. SL showed substantial, albeit temporally graded retrograde memory deficits on the adapted AI. Strikingly, whilst SL could not provide any specific details of events from the past two weeks or past two years and had impaired recall of events from her late 30s, her descriptions of potential future events were normal in number of event details and plausibility.

Conclusions: This dissociation of past and future events performance after mammillary body and fornix damage is at odds with the findings of the majority of patients with adult-onset hippocampal amnesia. It suggests that these nonhippocampal regions of the Papez circuit are only critical for past event retrieval and not for the generation of possible future events. 
Into the future with little past

Keywords: thalamus, Wernicke-Korsakoff's, diencephalic lesion, cognition, memory 
Into the future with little past

\section{Introduction}

Tulving (2002) described episodic memory as the ability to conjure personally experienced events of a known temporality that are rich in contextual details. Recently, this skill has been linked to the ability to mentally pre-experience possible personal future events, termed episodic future thinking (Atance \& O'Neill, 2001), at the behavioural and neurological level (for a recent review see Addis \& Schacter, 2012)

In healthy participants, studies have demonstrated similarities in the phenomenological detail of personal past and future event descriptions (Addis \& Schacter, 2008; D'Argembeau \& Van der Linden, 2004; Hassabis, Kumaran, Vann, \& Maguire, 2007; Levine et al., 1998; Levine, Svoboda, Hay, Winocur, \& Moscovitch, 2002; Stuss, 1991). For example, as temporal distance to the present increases, the amount of episodic details (Addis \& Schacter, 2008), and vividness (D'Argembeau \& Van der Linden, 2004) drops in both past and future event descriptions. The behavioural similarities between past and future events have been further corroborated by findings of shared neural correlates across the two processes in functional imaging experiments (Buckner \& Carroll, 2007; Okuda et al., 2003; Szpunar, Watson, \& McDermott, 2007). Neuroimaging studies involving both tasks reveal activations of a core brain network, involving the medial and lateral temporal lobe, lateral parietal, medial prefrontal and posterior cingulate/retrosplenial regions (Schacter \& Addis, 2007; Schacter, Addis, \& Buckner, 2007).

Studies involving patients with adult onset medial temporal lesions have provided evidence of the hippocampus' importance in past and future thinking. Of the 22 patients reported in the literature with adult-onset anterograde and retrograde 
Into the future with little past

amnesia caused by bilateral medial temporal lesions, 15 have shown significant problems describing both past events and possible future ones: (MC in Andelman, Hoofien, Goldberg, Aizenstein \& Neufeld, 2010; 4 out of 5 cases in Hassabis et al., 2007; DB in Klein, Loftus \& Kihlstrom, 2002; 8 patients in Race, Keane \& Verfaelli, 2011; NN later named KC in Tulving, 1985 and Rosenbaum et al., 2005), but seven have not (1 out of 5 cases (PO1) in Hassabis et al., 2007; 6 cases, albeit with intact recall of remote events, in Squire et al., 2010). The Adapted Autobiographical Inventory (Addis \& Schacter, 2008) was created to explore past and future thinking using the same technique and matching time intervals in both temporal directions. This has been used, for example, to examine changes in past and future thinking in older versus younger subjects.

Based on this behavioural and neuroanatomical evidence, Schacter and Addis (2007) proposed the constructive episodic simulation hypothesis; episodic future thinking is achieved through the flexible recombination and integration of (past) episodic memory traces into novel events via relational processes. According to Schacter and Addis (2007), conjuring potential new experiences draws upon various features of encoded experiences, including outputs from perceptual systems and interpretation by semantic systems, in addition to the episodic memory network. Hence activation of a large-scale brain system, which depends heavily on the hippocampus for relational processes used during the recombining process (Schacter \& Addis, 2007; Schacter, Addis, \& Buckner, 2007), seems to be crucial for both the ability to retrieve personally experienced past events and episodic future thinking.

It should be noted that the hippocampus is only the first relay station of the episodic memory or Papez circuit, which encompasses the hippocampus, fornix, mammillary bodies, thalamus and anterior cingulate (see Carlesimo, Lombardi \& 
Into the future with little past

Caltagirone, 2011 for a review). Studies in both humans and animals have found that damage to any of the relay structures involved in this circuit results in episodic memory retrieval deficits (for a review see Aggleton \& Brown, 1999). Given the association between deficient past and future thinking found in patients with damage in other parts of the circuit (namely the hippocampus; Andelman et al., 2010; Hassabis et al., 2007; Klein et al., 2002; Race et al., 2011; Rosenbaum et al., 2005; Tulving, 1985), we sought to determine whether the same pattern would be observed when the hippocampus is intact but other Papez structures are damaged.

Patients with Wernicke-Korsakoff's syndrome (WKS) provide an ideal human lesion model to test this notion, as neuropathological studies have consistently found atrophy in the mammillary bodies (Sheedy, Lara, Garrick, \& Harper, 1999) and occasionally in the anterior thalamic nuclei (Harding, Halliday, Caine, \& Kril, 2000). Atrophy in these brain regions is associated with an amnestic presentation (Jung, Chanraud, \& Sullivan, 2012) and/or selective deficits in contextual memory retrieval (Tsivilis et al., 2008; Vann, 2010; Vann et al., 2009). Patients with WKS also show severe deficits in the recall of past events (i.e., autobiographical memory), with most patients having better recall of remote memories than recent ones (Kopelman, 1989; Kopelman, Stanhope, \& Kingsley, 1999). Some patients with WKS confabulate (produce false statements) about both the past and the future (i.e., La Corte, Serra, Attali, Boisse \& Dalla Barba, 2010), typically spontaneously (Schnider, 2008). In two published cases, confabulation related only to future events (Burgess \& McNeil, 1999; Schnider et al., 2013). In both cases, confabulations were recurrent, spontaneous and fixed: concerned an obligation to which they felt a need to respond. The obligation was related to a specific episode from the past, but was not relevant for the future. To our knowledge, no study to date has investigated whether ability to 
Into the future with little past

construct relevant future events on demand is also impaired in WKS patients with selective mammillary body damage who present with anterograde and temporally limited retrograde amnesia.

The current study aims to fill this gap by investigating the diencephalic contribution to episodic future thinking in a case with WKS amnesia (SL).

Importantly, SL showed selective damage to the mammillary bodies and fornix, while the anterior thalamus and hippocampus remained relatively intact. Furthermore, we employed a structured, detailed approach to contrast past and future thinking: the adapted Autobiographical Interview (Addis \& Schacter, 2008). Given the behavioural similarities between past and future thinking found in healthy participants and in patients with bilateral mesial temporal lesions we predicted that SL would show impaired future thinking due to her striking deficits in episodic memory.

\section{Method}

Approvals were granted by the Royal Prince Alfred Hospital, University of Sydney (Protocol No X09-0338 \& HREC/09/RPAH/564) and University of New South Wales (Biomedical, ref. \#10035) Human Research Ethics Committees. All participants provided informed consent.

\subsection{Case Description}

SL was a 43-year-old, right-handed woman who presented in February 2009 with a 10-day history of increasing ataxic gait, blurred vision, confusion and amnesia. She was diagnosed with Wernicke Korsakoff's encephalopathy caused by nutritional deficiency and high alcohol consumption (2 litres of wine per day for 7 years). Treatment was intravenous thiamine and withdrawal of alcohol. Her initial MRI as well as a later one, from December 2009 confirmed diffuse cerebral and cerebellar 
Into the future with little past

atrophy as well as hyperintensities bilaterally in the mamillary bodies and periaqueductal grey. In the 2009 MRI, atrophy is visible in this region (Figure 1). A region of interest volumetric analysis was conducted on SL's fornix, mammillary bodies, thalami and hippocampi, and compared to a control group $(n=15)$ of similar mean age $(M=48.3 ; S D=8.4)$. All volumetric analyses were conducted using the VBM and FIRST toolboxes in FSL (http://www.fmrib.ox.ac.uk/fsl/). The regions of interest (ROI) boundaries were established by masking the whole brain with the respective regions from the Harvard-Oxford probabilistic atlas and the Juelich Histological atlas. There is currently no anatomical ROI for the anterior thalamus for T1 scans, as the borders cannot be delineated on a macroscopic level. Instead, we used the first third of the anterior thalamus as a proxy regional measure, which we have also used in previous studies (Hornberger et al. 2011; Tan et al., 2013; Tu et al. 2015). All ROIs were corrected for total intracranial volume (TIV) to account for brain size differences across participants. SL had reduced mammillary body volumes $\left(\mathrm{mm}^{3}\right)[\mathrm{SL}=126$; controls: mean $=235(\mathrm{SD}=30.3)]$ and fornix volumes $\left(\mathrm{mm}^{3}\right)[\mathrm{SL}=$ 910; controls: mean $=1201(\mathrm{SD}=123.4)]$, but relatively intact anterior thalamus $\left(\mathrm{mm}^{3}\right)[\mathrm{SL}=3460 ;$ controls: mean $=2955(\mathrm{SD}=585.3)]$ and hippocampal volumes $\left(\mathrm{mm}^{3}\right)[\mathrm{SL}=7115$; controls: mean $=7579(\mathrm{SD}=424.1)]($ Figure 2$)$. Statistical analyses of the region-of-interest (ROI) volumes, employing single case statistics developed by J. R. Crawford and Garthwaite (2007), revealed significant volume changes for the mammillary bodies $(\mathrm{p}<0.01)$ and fornix $(\mathrm{p}<0.025)$ but not for the anterior thalamus $(\mathrm{p}>0.1)$ and hippocampus $(\mathrm{p}>0.1)$.

----- Figure 1 \& Figure 2-----

From the time of her admission, SL's clinical presentation was notable for her dense anterograde and retrograde amnesia. For example, during her initial illness, she 
Into the future with little past

had no recollection of her current partner of several years, but instead thought that she was still living in London with her ex-husband. According to the history provided by a relative, this would have been her situation 16 years earlier. In addition, she showed no day-to-day memory for events. On follow up conducted a year after the initial admission she was sober. She came to realise who her current partner was. She had not returned to work, but had a volunteer role in a charity shop which she attended a few times per week. Each time she went there as for the first time. One problem that arose as a result of her ongoing poor memory was a great accumulation in second hand clothes that she brought home because she had forgotten her previous purchases.

SL had completed a Bachelor's degree in Economics (15 years of education) and had worked in the related field for some years after graduating. She had not worked, however, for several years prior to admission. She had no other known preexisting history of psychiatric or neurological disorders. When she was tested, she was taking oral Thiamine and was no longer drinking alcohol. A mood inventory (Depression, Anxiety Stress Scales; Lovibond \& Lovibond, 1995) indicated no symptoms of stress, anxiety or depression.

Results from SL's standard neuropsychological assessment (all carried out $\geq 9$ months post onset) are shown in Table 1. Relative to normative data, SL demonstrated severely impaired anterograde memory on tests involving verbal (Verbal Paired Associates from the Wechsler Memory Scale - Third Edition, Wechsler, 1997b; Rey Auditory Verbal Learning Test, Lesak, 1976) or visual (Rey Complex Figure Test; Rey, 1941) material: delayed recall $\leq 5^{\text {th }}$ percentile in all instances. In addition, SL's generativity/verbal fluency was also severely impaired, scoring in the bottom $1^{\text {st }}$ percentile for the Controlled Oral Word Association Test (Benton \& Hamsher, 1976). Nevertheless, she scored within the age-appropriate range for all other measures of 
Into the future with little past

executive functioning tested: working memory (Digit Span Backward from the Wechsler Adult Intelligence Scale -Third Edition; Wechsler, 1997a), mental flexibility (Trail Making Test - B; Partington \& Leiter, 1949), response initiation and suppression (Hayling Test; Burgess \& Shallice, 1997) and visuospatial reasoning (BrixtonTest; Burgess \& Shallice, 1997). The test of past and future thinking (as described in the next section) was administered in July 2010.

----- Table 1 -----

Materials

First, a procedure used by Addis et al. (2008) was employed to investigate recall of past and construction of future autobiographical events. Narratives were scored using a scoring procedure for the Autobiographical Interview developed by Levine and colleagues (2002). Participants were asked to recall or imagine in as much detail as possible four specific events from each of the following time periods: past two years (excluding last month), past two weeks, next two weeks and next two years (excluding next month), each within a three-minute time limit, including general probing. Immediately after completion of each event description, participants dated and rated it for phenomenological detail on (i) vividness ( $1=$ vague to $6=$ extremely clear/as if it is happening now), (ii) how the event affected them emotionally ( $1=$ no change in how I felt to $6=$ tremendous emotional change) and (iii) how personally significant the event was to them $(1=$ no importance at all to $6=$ of great importance $)$.

The order of Direction (past, future) was counterbalanced across control participants. While five participants recalled the past first, six participants recalled the future first. However, in both temporal directions (past or future) participants were asked to recall proximal (two weeks) before distal (two years) events to reduce 
cognitive load. SL was asked to recall past events first and future events second. Mann Whitney U test showed that the order or recall did not affect neither the number of internal (past weeks, $p=.33$, past years, $p=.66$; future weeks, $p=.54$, future years, $p=.23$ ) and external (past weeks, $p=.43$, past years, $p=.43$; future weeks, $p=.18$, future years, $p=.43$ ) details generated nor the phenomenological ratings: vividness (past weeks, $p=1.00$, past years, $p=.33$; future weeks, $p=.93$, future years, $p=.54$ ), emotional impact (past weeks, $p=.93$, past years, $p=.79$; future weeks, $p=.54$, future years $p=.06$ ) and personal significance (past weeks, $p=.08$, past years, $p=.25$; future weeks, $p=.25$; future years, $\mathrm{p}=.25$ )

Second, the original Autobiographical Interview (AI, Levine et al., 2002) was used to examine the temporal extent of SL's autobiographical memory deficits. Participants were asked to recall one specific event from each of four life periods: Teenage Years (11-17 years), Early Adulthood (18-35), Middle Adulthood (36-one year prior to assessment) and Recent Time (last year). On completion of free recall of each event a general probe was given.

Both interviews were audio taped, transcribed verbatim and scored using the AI manual. The AI separates details that are central to the episode (internal/episodic) from details that are episode non-specific (external/semantic). In addition, it distinguishes types of internal (event, time, place, perceptual, and emotion/thought) and external [semantic, repetitions and other (such as metacognitions or editorilizations)] details. Total recall scores (free recall + recall after general probe) of internal and external as well as different types of details were obtained for each period in the adapted and original Autobiographical Interviews. All transcripts of the first interview were scored by the main investigator (JT) who was trained in scoring. Ten randomly selected past and future narratives were scored by another independent, 
Into the future with little past

trained researcher. The intraclass correlations were high for internal $($ ICC $=.89, p$ $<.01)$ and external $(I C C=.83, p<.01)$ details. The transcripts of the second interview were first segmented into informational bits or details, classified as a unique occurrence, observation or thought, typically expressed as a grammatical clause. Each detail was then categorised as "internal", or" external', representing episodic and semantic memory, respectively. Here, we focused on the retrieval of internal (i.e., episodic) details as an index of overall autobiographical event recollection. Internal details were those details relating directly to the main episode, located within a specific spatiotemporal context, and reflected episodic re-experiencing. All scoring was performed by a trained scorer $(\mathrm{MH})$ who was trained on an independent set of stories with high rating consistency $(\mathrm{ICC}=.91, \mathrm{p}<.01) . \ldots .$. Michael/Sicong????...

Finally, phenomenological qualities of events recalled in the first interview were also rated by two independent raters blind to the group membership (SL vs Controls) when a significant difference was found between SL's and control participants' ratings of events phenomenological qualities. To further quantify the scene construction ability, which was not rated by study participants but is of theoretical significance, all transcripts of events from the first interview were also rated for contiguity $(1=$ the event was a collection of separate images to $6=$ I could see the event unfolding in front of my eyes) by the same two independent raters. The mean ratings were used in the analyses.

Statistical analyses

First, SL scores were compared to scores obtained by the control group using the modified t-tests (J. R. Crawford \& Howell, 1998) with one-tail (as SL scores were expected to be below the scores of the controls) and alpha at .05. We used the Singlims program, specifically developed to assess whether single case scores differ 
Into the future with little past

significantly from a control sample (J.R. Crawford \& Garthwaite, 2002). Second, the differences in scores obtained in recall of past and generation of future event details was compared using the DiffBayes.exe program (J. R. Crawford \& Garthwaite, 2007). This program, based on Bayesian approach, was developed to compare differences between scores on two tasks obtained by a single case with the differences between scores obtained on the same two tasks obtained by a control group. It provides: probability ( $\mathrm{p}$ value) and estimate of the "percentage of the control population that would exhibit a more extreme discrepancy" (Crawford \& Garthwaite, 2007, p 365) than a single case. Both programs were accessed through Professor Crawford's webpage: http://homepages.abdn.ac.uk/j.crawford/pages/dept/psychom.htm.

\section{Results}

Past and future thinking: Adapted Autobiographical Interview

As can be seen in Figure 3, SL recalled no specific events from the past two weeks or past two years. Modified t-tests showed that her scores $(\mathrm{RSs}=0)$ differed significantly from controls $[\mathrm{n}=11$, comparable in age $\mathrm{M}=48.27$ years $(\mathrm{SD}=6.65)$ and education $\mathrm{M}=16.18$ years $(\mathrm{SD}=1.99)$ ], for both internal and external details provided in specific events that happened over the (i) past two weeks $[t(10)=-3.69, p=0.002, Z$ $C C=-3.857$ and $t(10)=-2.57, p=0.014, Z-C C=-2.680$ respectively] and (ii) past two years $[t(10)=-4.84, \mathrm{p}<0.001, Z-C C=-5.053$ and $t(10)=-2.93, p=0.008, Z-C C=-$ 3.062, respectively].

In contrast, SL generated specific future events. The number of internal and external details provided by SL and control participants did not differ [next few weeks $(t(10)=-$ $0.37, p=.179, Z-C C=-0.389$ and $t(10)=-1.04, p=0.081, Z-C C=-1.083$ respectively), next few years $(t(10)=-0.53, p=0.151, Z-C C=-0.556$ and $t(10)=-0.98, p=0.087, Z-C C$ $=-1.027$ respectively)]. Moreover, the Bayesian tests revealed that the difference 
Into the future with little past

between past and future internal (but not external) details was significantly greater for SL relative to controls $(p<.001 ; Z-D D=-4.759$ and $p=.368 ; Z-D D=-0.403)$. The likelihood that the difference between the number of past and future internal details for a control participant would be greater than that of SL was 0.00079 .

----- Figure 3 -----

Example transcripts of past and future proximal and distal event descriptions provided by SL and by one of the control participants are given below (Figure 4 a) and b), respectively). As can be seen, SL's description of a future holiday event was detailed, vivid and well organised, but she cannot recall any specific events from the past two years. There was no evidence of confabulation in the events she generated, all were considered plausible.

----- Figure 4 -----

Analysis of the phenomenological details was not conducted for past events, as SL had not recalled any information. As can be seen in Table 2, SL's ratings of future events provided for the next two weeks and next two years were similar to ratings provided by control subjects in terms of (i) emotional impact and (ii) personal significance. Similarly, there was no significant difference between SL and control subjects in their ratings of the vividness of distal future events. However, compared to the control group, SL rated her proximal future event descriptions (next two weeks) as being of significantly lower vividness. Next, two research staff members (blinded to the participants' group membership) rated the vividness and contiguity of the transcribed future events provided by SL and control participants. Vividness and 
Into the future with little past

contiguity ratings of SL's proximal and distant future events made by the research staff were comparable to that of control participants.

----- Table 2 -----

3.2 Memory for the past: Autobiographical Interview

As shown in Figure 5, SL provided a significantly smaller number of internal details from the more recent (middle age: $t(17)=-3.21, p=0.003, Z-C C=-3.294$ and last year: $t(17)=-3.53, p=0.001, Z-C C=-4.301)$, but not distant (teenage $t(17)=0.42, p=0.340$, $Z-C C=0.428$ and early adult $t(17)=0.70, p=0.247, Z-C C=0.717)$ life periods relative to a group of control subjects of similar age and education [n=18; age $M=45.3$ (SD =4.9); education $\mathrm{M}=12.8(\mathrm{SD}=3.4)]$. SL and control subjects generated comparable numbers of external details across the three earlier life periods: teenage $(t(17)=0.35$, $p=0.364, Z-C C=0.362)$, early adult $(t(17)=-0.24, p=0.405, Z-C C=-0.250)$ and middle age $(t(17)=1.40, p=0.089, Z-C C=1.441)$ Similar to the findings on the adapted AI, for the most recent year, SL's recall of external details from an event was impaired $(t(17)=-1.84, p=0.04, Z-C C=-1.889)$.

----- Figure 5 -----

\section{Discussion}

To our knowledge, this is the first study that examined future thinking in a patient (SL) with severe WKS amnesia and volumetric confirmation of bilateral mammillary body damage with relatively preserved hippocampi and anterior thalamic nuclei. Although SL had substantial (albeit temporally limited and temporally graded) deficits in episodic memory in general, she was able to imagine fictitious, but plausible, future experiences of comparable richness and with similar 
Into the future with little past

phenomenological qualities to those of controls. This was at odds with our prediction and with the findings for the majority of patients with adult-onset amnesia secondary to medial temporal lesions.

Unlike all other amnesia cases with selective lesions of the Papez circuit in which future thinking has been investigated, SL had only limited hippocampal atrophy, but instead bilateral mammillary body and fornix damage. To date, the only other study that examined past and future thinking in patients with diencephalic damage involved two stroke cases with lesions of the medial-dorsal thalamus (Weiler, Suchan, Koch, Schwarz, \& Daum, 2011). The patients in that study were free of anterograde memory deficits, had intact recall of events from the past and showed only very mild deficits in future thinking. The discrepancy between our findings and the results of Weiler and colleagues can be attributed to the differences in the diencephalic structures involved and their connectivity. Patients with lesions involving the medial-dorsal thalamus were specifically selected by Weiler et al. (2011) because of the strong connection this nucleus has to the prefrontal cortex (Johansen-Berg et al., 2005). The medial-dorsal thalamus does not receive any direct afferents from the hippocampus. Thus it is not surprising that the two patients examined by Weiler and colleagues showed deficits in executive skills, but only very mild anterograde memory deficits. By contrast, SL's lesions in the mammillary bodies and fornix, which receive their main input fibres from the hippocampus (Aggleton et al., 2010; Vann, 2010), produced a severe amnestic syndrome, but little deficits in executive skills. Thus, the divergent findings of the two studies with respect to future thinking are likely explained by the difference in the anatomical connectivity of the lesioned regions and the opposite pattern of preserved and impaired cognitive skills. It seems likely that damage to the links between the thalamus and frontal cortices (and 
Into the future with little past

associated deficits in executive skills) as a result of stroke in the medial-dorsal nucleus may have resulted in the subtle deficits in future thinking reported in the cases reported by Weiler et al. (2011). In contrast, relatively preserved executive skills could have supported SL's ability to generate future events by utilising established event-scripts stored in semantic memory or from the preserved, distant events.

The discrepancy between SL's past recall deficits and relatively intact future thinking was striking. Such discrepancy is typically not found in patients with adult onset anterograde and retrograde amnesia, when amnesia is secondary to bilateral medial temporal lesions (MC in Andelman et al., 2010; 4 out of 5 cases in Hassabis et al., 2007; DB in Klein, Loftus \& Kihlstrom, 2002; 8 patients in Race, Keane \& Verfaelli, 2011; NN later named KC in Tulving, 1985 and Rosenbaum et al., 2005). Although Squire et al. (2010) and Hassabis et al. (case PO1, 2007) reported a pattern similar to SL's in several cases with adult onset amnesia, in these cases, the reduction in hippocampal volume was extreme (i.e., in excess of 3 standard deviations from the control mean in the case reported by Squire et al., 2010 and greater than $40 \%$ in Hassabis et al case PO1. In SL, bilateral hippocampal volume was slightly (1.09 standard deviations), but not significantly, reduced relative to controls. While this raises a possibility that SL experienced some hippocampal volume loss as a result of her illness, the severity of her memory deficits greatly exceeds memory deficits that are likely to arise form a possible mild reduction in hippocampal volume. Instead, her memory deficits are attributable to the significant volume changes evident in her mammillary bodies and fornix. Although an association between amnesia and mammillary body lesions has been known for a long time (Gudden, 1896), the contribution of this diencephalic structure to memory is still being discussed (Vann, 2010) and the specific contributions that the mammillary bodies make to future 
Into the future with little past

thinking had not been previously investigated. Their small size makes it difficult to detect significant activations with fMRI. Indeed, the mammillary bodies are often seen merely as a relay station between the hippocampus and the anterior thalamus, as they do not comprise interneurons, which usually allow excitatory or inhibitory modulation of neural signals (Aggleton et al., 2010; Vann, 2010). Still, the medial nuclei of the mammillary bodies, which are always affected in WKS (Kopelman, 1995), receive input from multiple regions (i.e., hippocampus, tegmentum, prefrontal cortex). Convergence of these inputs appears to be critical for intact retrieval of contextual-rich information (see also Tsivilis et al., 2008). Our findings confirm that the mammillary bodies and fornix are critical for retrieval of contextually rich autobiographical memories, but they play little role in future thinking. This contrasts with the role of the hippocampus, which seems to be involved in both processes (Andelman et al., 2010; Hassabis et al., 2007; Klein et al., 2002; Race et al., 2011; Rosenbaum et al., 2005; Tulving, 1985; though see Squire et al., 2010 for some cases of adult-onset hippocampal amnesia without deficits in future thinking). These findings imply that both past and future event thinking share only a subset of brain regions, including the hippocampus, and although past event retrieval requires additional processing in diencephalic brain regions, future thinking does not.

The dissociation of past and future thinking that we observed in our case study seems to contradict the constructive episodic simulation hypothesis (Schacter \& Addis, 2007; Schacter, Addis, \& Buckner, 2007) and findings that the amount of episodic details (Addis \& Schacter, 2008) drops in both past and future event descriptions as temporal distance from the presence increases. Indeed, according to this hypothesis, there should be a clear correlation between recalled details of past events and the generation of future events, but instead, we found a severe impairment 
Into the future with little past

limited to the description of past events. Not only were SL's descriptions of future events comparable to those of $\mathrm{NC}$ subjects in terms of quantity of internal and external details, although her scores are somewhat below the mean scores of control subjects, but also in most of the phenomenological ratings. Only SL's self-ratings of vividness of proximal future memories were lower than those of control subjects. The transcripts of these events were rated (by an independent judge) as being of comparable vividness as the events described by control subjects. Thus the significance of SL's lower rating of vividness of her proximal future event is unclear, but will be considered in our future studies. Her ratings of the emotional impact and personal significance of the future events generated fell within normal limits.

Previous findings in WKS patients indicate that some very remote autobiographical memories remain intact (Kopelman, 1989; Kopelman et al., 1999), which is consistent with our findings of preserved episodic memories for teenage and early adulthood years in SL. It is possible that when creating future scenarios, SL utilised distant, preserved episodic memories to compensate for the episodic memory loss that covered some 15-20 years of her life, an issue we are currently investigating. This is further corroborated by her clinical notes which showed that her episodic present time was situated well and truly in the past. We propose that lesions in non-hippocampal regions of the Papez circuit regions do not affect the simulation of future events, because patients can either rely on the retrieval of very remote autobiographical memories and/or utilise established event-scripts stored in semantic memory as scaffoldings to create future events.

It will be critical to corroborate these findings in a bigger sample of patients in the future. Case studies are often viewed as controversial as it is not clear whether the examined patient is representative for effects on a population level. 
Into the future with little past

Nevertheless, our findings replicate previous autobiographical memory findings in patients with WKS and the neuroimaging profile was very clear cut, which suggests that the findings in this study are genuine. Taken together, our results suggest that mammillary body and fornix damage specifically impaired anterograde memory and recall of autobiographical memories from the past several years, but not projections of future events. This dissociation suggests that the hippocampus may be critical for both processes while non-hippocampal diencephalic structures are only involved in the retrieval of the past. From the cognitive perspective, dissociation between past and future events found in our study, but also in two other studies that involved patients with anterograde amnesia and retrograde memory deficits (temporally extensive, PO1 in Hassabis et al., 2007 and temporally graded, 6 cases in Squire et al., 2010) are at odds with the constructive episodic hypothesis, which suggests that construction of future events involves a single cognitive route: recollection of past events from which episodic details are sourced and recombined to construct future events via relational processes (i.e., relational memory). Thus we purport that an alternative cognitive and anatomical route enables patients with impairments in relational memory and autobiographical episodic memory to imagine and describe future events. In particular, preserved executive skills might allow one to draw on event-scripts stored in semantic memory to construct future events. Anatomically, a potential split of preversus post-commissural fornix projections from the hippocampus could explain the dissociation of the functions. More specifically, only post-commissural fornix fibers project to the mammillary bodies whereas pre-commissural fibres project to medial prefrontal cortex regions (Vann \& Aggleton, 2004), which has been shown to be critically involved in future thinking (Stawarczyk \& D'Argembeau, 2015). Hence it is possible that SL was able to construct future events because her hippocampus and 
Into the future with little past

frontal lobes were still able to work together even though (because of a mammillary body lesion) she was unable to form new relational memories. Further experimental studies examining dissociations and exceptional cases in general as well as clinical populations are likely to provide further insight into cognitive architecture of future thinking, and refine theories.

\section{Acknowledgements}

MH was supported by Alzheimer Research UK, the Wellcome Trust and the Newton Trust. Suncica Lah was in part supported by The University of Sydney Thompson Fellowship. ST is supported by Alzheimer's Australia Dementia Research Foundation and NHMRC of Australia awards. The research was also supported by an ARC Centre of Excellence grant into Cognition and Its Disorders. We would like to thank Carly Black and Michael Gascoigne for rating of transcripts and Hayley Donohue for assistance in manuscript preparation.

\section{Disclosure}

No financial interest or benefits have been gained or are expected to be gained from the applications of this research. 
Into the future with little past

\section{Figure Legends}

Figure 1. Magnetic Resonance Imaging (MRI) scan showing SL's lesions in the mammillary bodies compared to a healthy control.

Figure 2. Regions of interest (hippocampus, fornix, mammillary bodies, anterior thalamus) volumes in SL and controls. Line indicates mean volume in controls.

Figure 3. Mean number of internal and external event details for SL and controls for adapted AI divided with respect to temporal distance (weeks, years) and direction (past, future). Error bars represent standard error of the mean.

Figure 4. Transcripts of proximal (a) and distal (b) past and future event descriptions provided by SL and a control participant on the adapted AI.

Figure 5. Mean number of internal and external event details for SL and controls obtained on AI. Error bars represent standard error of the mean. 
Into the future with little past

\section{References}

Addis, D. R., \& Schacter, D. L. (2008). Constructive episodic simulation: Temporal distance and detail of past and future events modulate hippocampal engagement. Hippocampus, 18(2), 227-237. doi: 10.1002/hipo.20405

Addis, D. R., \& Schacter, D. L. (2012). The hippocampus and imagining the future: Where do we stand? Frontiers in Human Neuroscience, 5. doi: 10.3389/fnhum.2011.00173

Aggleton, J. P., \& Brown, M. W. (1999). Episodic memory, amnesia, and the hippocampal-anterior thalamic axis. The Behavioral and Brain Sciences, 22(3), 425-489. doi: 10.1017/S0140525X99002034

Aggleton, J. P., O'Mara, S. M., Vann, S. D., Wright, N. F., Tsanov, M., \& Erichsen, J. T. (2010). Hippocampal-anterior thalamic pathways for memory: Uncovering a network of direct and indirect actions. The European Journal of Neuroscience, 31(12), 2292-2307. doi: 10.1111/j.1460-9568.2010.07251.x

Andelman, F., Hoofien, D., Goldberg, I., Aizenstein, O., \& Neufeld, M. Y. (2010). Bilateral hippocampal lesion and a selective impairment of the ability for mental time travel. Neurocase, 16(5), 426-435. doi:

$10.1080 / 13554791003623318$

Atance, C. M., \& O'Neill, D. K. (2001). Episodic future thinking. Trends in Cognitive Sciences, 5(12), 533-539. doi: 10.1016/S1364-6613(00)01804-0

Benton, A. L., \& Hamsher, K. (1976). Multilingual Aphasia Examination. Iowa City: University of Iowa.

Buckner, R. L., \& Carroll, D. C. (2007). Self-projection and the brain. Trends in Cognitive Sciences, 11(2), 49-57. doi: 10.1016/j.tics.2006.11.004 
Into the future with little past

Burgess, P. W., \& McNeil, J. E. (1999). Content-specific confabulation. Cortex; $a$ Journal Devoted to the Study of the Nervous System and Behavior, 35(2), 163182. doi: 10.1016/S0010-9452(08)70792-5

Burgess, P. W., \& Shallice, T. (1997). The Hayling and Brixton Tests: Manual. Suffolk, England: Thames Valley Test Company Limited.

Carlesimo, G. A., Lombardi, M. G., \& Caltagirone, C. (2011). Vascular thalamic amnesia: A reappraisal. Neuropsychologia, 49(5), 777-789. doi: 10.1016/j.neuropsychologia.2011.01.026

Crawford, J. R., \& Garthwaite, P. H. (2002). Investigation of the single case in neuropsychology: Confidence limits on the abnormality of test scores and test score differences. Neuropsychologia, 40, 1196-1120.

Crawford, J. R., \& Garthwaite, P. H. (2007). Comparison of a single case to a control or normative sample in neuropsychology: Development of a bayesian approach. Cognitive Neuropsychology, 24(4), 343-372. doi:

$10.1080 / 02643290701290146$

Crawford, J. R., \& Howell, D. C. (1998). Comparing an individual's test score against norms derived from small samples. The Clinical Neuropsychologist, 12(4), 482-486. doi: 10.1076/clin.12.4.482.7241

D'Argembeau, A., \& Van der Linden, M. (2004). Phenomenal characteristics associated with projecting oneself back into the past and forward into the future: Influence of valence and temporal distance. Consciousness and Cognition, 13(4), 844-858. doi: 10.1016/j.concog.2004.07.007

Gudden, H. (1896). Klinische und anatommische beitrage zur kenntnis der multiplen alkoholneuritis nebst bemerzungen uber die regenerationsvorgange im peripheren nervensystem. Archiv fur Psychiatrie, 28(3), 643-741. 
Into the future with little past

Harding, A., Halliday, G., Caine, D., \& Kril, J. (2000). Degeneration of anterior thalamic nuclei differentiates alcoholics with amnesia. Brain: A Journal of Neurology, 123(Pt 1), 141-154. doi: 10.1093/brain/123.1.141

Hassabis, D., Kumaran, D., Vann, S. D., \& Maguire, E. A. (2007). Patients with hippocampal amnesia cannot imagine new experiences. Proceedings of the National Academy of Sciences of the United States of America, 104(5), 17261731. doi: 10.1073/pnas.0610561104

Johansen-Berg, H., Behrens, T. E., Sillery, E., Ciccarelli, O., Thompson, A. J., Smith, S. M., \& Matthews, P. M. (2005). Functional-anatomical validation and individual variation of diffusion tractography-based segmentation of the human thalamus. Cerebral Cortex, 15(1), 31-39. doi: 10.1093/cercor/bhh105

Jung, Y. C., Chanraud, S., \& Sullivan, E. V. (2012). Neuroimaging of Wernicke's encephalopathy and Korsakoff's syndrome. Neuropsychology Review, 22(2), 170-180. doi: 10.1007/s11065-012-9203-4

Klein, S. B., Loftus, J., \& Kihlstrom, J. F. (2002). Memory and temporal experience: The effects of episodic memory loss on an amnesic patient's ability to remember the past and imagine the future. Social Cognition, 20(5), 353-379. doi: $10.1521 /$ soco.20.5.353.21125

Kopelman, M. D. (1989). Remote and autobiographical memory, temporal context memory and frontal atrophy in Korsakoff and Alzheimer patients. Neuropsychologia, 27(4), 437-460. doi: 10.1016/0028-3932(89)90050-X

Kopelman, M. D. (1995). The Korsakoff syndrome. The British Journal of Psychiatry: The Journal of Mental Science, 166(2), 154-173. doi: 10.1192/bjp.166.2.154 
Into the future with little past

Kopelman, M. D., Stanhope, N., \& Kingsley, D. (1999). Retrograde amnesia in patients with diencephalic, temporal lobe or frontal lesions. Neuropsychologia, 37(8), 939-958. doi: 10.1016/S0028-3932(98)00143-2

La Corte, V., Serra, M., Attali, E., Boisse, M. F., \& Dalla Barba, G. (2010). Confabulation in Alzheimer's disease and amnesia: A qualitative account and a new taxonomy. Journal of the International Neuropsychological Society, 16(6), 967-974. doi: 10.1017/S1355617710001001

Levine, B., Black, S. E., Cabeza, R., Sinden, M., Mcintosh, A. R., Toth, J. P., . . . Stuss, D. T. (1998). Episodic memory and the self in a case of isolated retrograde amnesia. Brain, 121(10), 1951-1973. doi: 10.1093/brain/121.10.1951

Levine, B., Svoboda, E., Hay, J. F., Winocur, G., \& Moscovitch, M. (2002). Aging and autobiographical memory: Dissociating episodic from semantic retrieval. Psychology and Aging, 17(4), 677-689. doi: 10.1037/0882-7974.17.4.677

Lezak, M. (1976). Neuropsychological Assessment. New York, NY: Oxford University Press.

Lovibond, P. F., \& Lovibond, S. H. (1995). The structure of negative emotional states: Comparison of the Depression Anxiety Stress Scales (DASS) with the Beck Depression and Anxiety Inventories. Behaviour Research and Therapy, 33(3), 335-343. doi: 10.1016/0005-7967(94)00075-U

Okuda, J., Fujii, T., Ohtake, H., Tsukiura, T., Tanji, K., Suzuki, K., . . Y Yamadori, A. (2003). Thinking of the future and past: The roles of the frontal pole and the medial temporal lobes. Neuroimage, 19(4), 1369-1380. doi: 10.1016/S10538119(03)00179-4 
Into the future with little past

Partington, J. E., \& Leiter, R. G. (1949). Partington's Pathway Test. The Psychological Service Centre Bulletin, 1, 9-20.

Race, E., Keane, M. M., \& Verfaellie, M. (2011). Medial temporal lobe damage causes deficits in episodic memory and episodic future thinking not attributable to deficits in narrative construction. The Journal of Neuroscience: The Official Journal of the Society for Neuroscience, 31(28), 10262-10269. doi: 10.1523/JNEUROSCI.1145-11.2011

Rey, A. (1941). L'examen psychologique dans les cas d'encephalopathie traumatique. Archives de Psychologie, 28, 215-285.

Rosenbaum, R. S., Köhler, S., Schacter, D. L., Moscovitch, M., Westmacott, R., Black, S. E., . . Tulving, E. (2005). The case of K.C.: Contributions of a memory-impaired person to memory theory. Neuropsychologia, 43(7), 9891021. doi: 10.1016/j.neuropsychologia.2004.10.007

Schacter, D. L., \& Addis, D. R. (2007). The cognitive neuroscience of constructive memory: Remembering the past and imagining the future. Philosophical Transactions of the Royal Society of London. Series B, Biological Sciences, 362(1481), 773-786. doi: 10.1098/rstb.2007.2087

Schacter, D. L., Addis, D. R., \& Buckner, R. L. (2007). Remembering the past to imagine the future: The prospective brain. Nature Reviews Neuroscience, 8(9), 657-661. doi: 10.1038/nrn2213

Schnider, A. (2008). The confabulating mind: How the brain creates reality. Oxford, UK: Oxford University Press.

Schnider, A., Nahum, L., Pignat, J. M., Leemann, B., Lovblad, K. O., Wissmeyer, M., \& Ptak, R. (2013). Isolated prospective confabulation in wernicke-korsakoff 
Into the future with little past

syndrome: A case for reality filtering. Neurocase, 19(1), 90-104. doi: $10.1080 / 13554794.2011 .654221$

Sheedy, D., Lara, A., Garrick, T., \& Harper, C. (1999). Size of mamillary bodies in health and disease: Useful measurements in neuroradiological diagnosis of wernicke's encephalopathy. Alcoholism, Clinical and Experimental Research, 23(10), 1624-1628. doi: 10.1111/j.1530-0277.1999.tb04053.x

Squire, L. R., van der Horst, A. S., McDuff, S. G., Frascino, J. C., Hopkins, R. O., \& Mauldin, K. N. (2010). Role of the hippocampus in remembering the past and imagining the future. Proceedings of the National Academy of Sciences of the United States of America, 107(44), 19044-19048. doi:

10.1073/pnas. 1014391107

Stawarczyk, D., \& D'Argembeau, A. (2015). Neural correlates of personal goal processing during episodic future thinking and mind-wandering: An ALE meta-analysis. Human Brain Mapping, 36(8), 2928 - 2947.

Stuss, D. T. (1991). Disturbance of self-awareness after frontal system damage. In G. P. Pritigano \& D. L. Schacter (Eds.), Awareness of Deficit After Brain Injury (pp. 63-83). Oxford, UK: Oxford University Press.

Szpunar, K. K., Watson, J. M., \& McDermott, K. B. (2007). Neural substrates of envisioning the future. Proceedings of the National Academy of Sciences of the United States of America, 104(2), 642-647. doi: 10.1073/pnas.0610082104

Tsivilis, D., Vann, S. D., Denby, C., Roberts, N., Mayes, A. R., Montaldi, D., \& Aggleton, J. P. (2008). A disproportionate role for the fornix and mammillary bodies in recall versus recognition memory. Nature Neuroscience, 11(7), 834842. doi: $10.1038 / \mathrm{nn} .2149$ 
Into the future with little past

Tulving, E. (1985). Memory and consciousness. Canadian Psychology/Psychologie Canadienne, 26(1), 1-12. doi: 10.1037/h0080017

Tulving, E. (2002). Episodic memory: From mind to brain. Annual Review of Psychology, 53, 1-25. doi: 10.1146/annurev.psych.53.100901.135114

Vann, S. D. (2010). Re-evaluating the role of the mammillary bodies in memory. Neuropsychologia, 48(8), 2316-2327. doi:

10.1016/j.neuropsychologia.2009.10.019

Vann, S. D., \& Aggleton, J. P. (2004). The mammillary bodies: two memory systems in one? Nature Review Neuroscience, 5(1), 35-44.

Vann, S. D., Tsivilis, D., Denby, C. E., Quamme, J. R., Yonelinas, A. P., Aggleton, J. P., . . Mayes, A. R. (2009). Impaired recollection but spared familiarity in patients with extended hippocampal system damage revealed by 3 convergent methods. Proceedings of the National Academy of Sciences of the United States of America, 106(13), 5442-5447. doi: 10.1073/pnas.0812097106

Wechsler, D. (1997a). Wechsler Adult Intelligence Scale -Third Edition. San Antonio, TX: The Psychological Corporation.

Wechsler, D. (1997b). Wechsler Memory Scale -Third Edition. San Antonio, TX: The Psychological Corporation.

Weiler, J., Suchan, B., Koch, B., Schwarz, M., \& Daum, I. (2011). Differential impairment of remembering the past and imagining novel events after thalamic lesions. Journal of Cognitive Neuroscience, 23(10), 3037-3051. doi: 10.1162/jocn.2011.21633 